\title{
Source localization in underwater sound fields
}

\author{
A. V. van den Berg ${ }^{1}$, R. J. A. Buwalda ${ }^{2}$ \\ 1 Fysiology I, Medical Faculty, Erasmus University Rotterdam, P.O. Box 1738, 3000 DR Rotterdam, The Netherlands \\ ${ }^{2}$ Laboratory of Comparative Physiology, Section Neuro-Ethology, Utrecht University, Padualaan 8, 3584 CH Utrecht, The Netherlands
}

Received: 1 July 1992/Accepted in revised form: 6 August 1993

\begin{abstract}
We investigated the acoustical information present in the field of arbitrary sound sources which may provide direction and distance to the source from a local reading of the sound field parameters. If the effects of reflections are negligible, the particle acceleration is directed radially at the instant of sound pressure nulls. The spectral relation between the radial component of the particle aceleration and the sound pressure is characterized by a critical frequency where a sharp transition occurs in the amplitude ratio and the phase relation of these variables. The critical frequency depends on the distance to the source and depends little on the source type (mono-, di- or quadrupole). Thus, a local reading of the particle acceleration and the sound pressure is in principle sufficient to localize the sound source in three dimensions. Fish might use this kind of information for acoustic orientation.
\end{abstract}

\section{Introduction}

The ability to localize a sound source has clearly survival value for a fish. Avoidance of a predator or capture of a prey is more likely to succeed the sooner it can be localized. Under water, sound fields carry in general more reliable information concerning the source location than e.g. visual or olfactory cues, especially at large distances. It seems appropriate then to consider sound source localization a major task for the fish if it attempts to monitor events in the distant surroundings.

Attraction and repulsion of fishes by sound sources is observed frequently. In many field studies it has been established that intermittent low-frequency noise is very effective for the attraction of sharks (Nelson 1965; Richard 1968; Myrberg et al. 1972; Nelson and Johnson 1972). Also, bony fish like the saithe and tuna can be attracted and repulsed by sound (Richard 1968; York 1972; Maniwa 1976; Olsen 1976) over large distances. Because usually only the aggregation of individuals near

Correspondence to: A. V. van den Berg the source is observed, these studies are inconclusive as to the manner in which the fishes orient towards the source. We do not know to what extent the directed responses are guided by the clearly visible tranducer. Nor is it clear from such experiments whether the fish use some sort of gradient search to find the source or are really able to perceive the direction towards the source 'on the spot'. However, in some instances, directed responses of shark towards the source have been reported at the onset of sound emission (Nelson 1965; Myrberg et al. 1972) up to distances of $200 \mathrm{~m}$ and over. Such observations strongly suggest that the fish perceives the sound source direction by a local reading of the soundfield. In addition, they suggest that the sensory system involved is the inner ear because the lateral line is not effectively stimulated at distances larger than a few body lengths from the source (Sand 1981).

Apart from the evidence gathered at sea from unconditioned responses of fish towards an active transducer, relevant data on piscine directional hearing have been obtained by psychophysical methods using carefully controlled soundfields in the laboratory or at sea. Most of these studies used gadoids or ostariophysians. Just noticeable differences (jnd) of sound source direction are usually not better than about $15^{\circ}$ (Chapman and Johnstone 1974; Hawkins and Sand 1977). The angular resolution depends on the signal to noise $(\mathrm{S} / \mathrm{N})$ ratio of the particle motion component (Buwalda 1981) and may increase to over $40^{\circ}$ for low $\mathrm{S} / \mathrm{N}$ ratios. Thus, directional discrimination requires accurate coding of the particle motion component. However, the particle motion at the position of the fish is identical for diametrically opposed sound sources, resulting in a $180^{\circ}$ ambiguity concerning the source direction if the fish is exclusively sensitive to particle motion. Nevertheless, some fish like the cod and the ide do discriminate opposite sound sources (Schuijf and Buwalda 1975; Buwalda et al. 1983). The mechanism involved appears to be a phase analysis between the sound pressure and the particle motion component (Schuijf and Buwalda 1975). Thus, a functional description of directional hearing by fish like the cod distinguishes two processes: (1) coding of the direction of the particle motion component and (2) a timing analysis 
between sound pressure and particle motion to resolve the $180^{\circ}$ ambiguity (Schuijf 1976). In this analysis the particle motion acts as a kind of pointer which is directed along the line connecting the fish and the sound source. The fish ear should merely measure the direction of the pointer. Obviously, the assumption is then that the particle motion is directed radially at any location in the sound field. This assumption is valid for monopoles which are characterized by volume changes (e.g. a pulsating air bubble).

Many natural sound sources are not monopole-like but rather more characterized by deformations without appreciable volume changes. The motion component in the fields of these sources is in general not radially directed (as in monopole fields) but contains tangential components as well (Harris 1964). Thus, the proposed mechanism of directional hearing, adequate as it may be for orienting towards a monopole, could be useless for the localization of many sound sources.

This raises the question of what type of directional information exists in the sound field when we drop the restriction to a monopole and also generalize to emission of sounds with arbitrary waveforms. We maintain, however, the restriction of approximate free-field conditions as we presently limit our attention to fish living in the open sea. Quite different cues for sound source localization may be expected in habitats where reflections dominate (Schuijf 1981). Here, we investigate if a temporal analysis of the relation between the local particle motion vector and sound pressure carries information concerning the source location with respect to the fish which is valid for any location in the sound field and holds irrespective of the source type. To anticipate, we will show that the local particle acceleration is radially directed at the instant of a sound pressure null at any position in the field where the sound pressure is non-vanishing.

\section{Directional information}

It may be instructive to start our analysis with a description of the properties of the field of a simple dipole. The field of this type of source describes adequately the field we may find around a sphere oscillating along an axis through its centre. Let the separation between the point sources of the acoustic doublet be $d$. The point of observation is characterized by the distance to the gravitational centre of the dipole $(r)$ and the angle $(\theta)$ with respect to the dipole axis. For a harmonic oscillation of the source (amplitude $A$; circular frequency $\omega$ ) the soundpressure at the point of observation is given by

$$
\begin{aligned}
p(r, \theta, t ; \omega)= & -\rho_{0} c A \cos (\theta)\left(\frac{j}{k r}+\frac{1}{(k r)^{2}}\right) \\
& \exp (j(\omega t-k r))
\end{aligned}
$$

where $k=2 \pi / \lambda$ [Lamb 1931; cf. Appendix, (A5), Kinsler et al. 1982], provided that the dipole length $(d)$ is small compared to the distance $(d \ll r)$ and also small in relation to the wavelength $(d \ll 1 / k)$. These approximations are well borne out for the low frequency range (up to $1 \mathrm{kHz}$ ) relevant to fish hearing. Using the linearized form of Euler's equation we may derive an expression for the particle acceleration.

$$
\begin{aligned}
\mathbf{a}(r, \theta, t ; \omega)= & -1 / \rho_{0} \nabla p(r, \theta, t ; \omega) \\
= & -1 / \rho_{0}[-((1+1 /(1+j k r)) / \\
& r+j k)=p(r, \theta, t ; \omega) \mathbf{e}_{r} \\
& \left.+(1 / r) \tan (\theta) p(r, \theta, t ; \omega) \mathbf{e}_{\theta}\right]
\end{aligned}
$$

The unit vectors $\left(\mathbf{e}_{r}, \mathbf{e}_{\theta}\right)$ denote the (outward) radial and the tangential direction (of increasing $\theta$ ) at the location $(r, \theta)$ in the sound field.

For a given position of the observer ( $r, \theta$ fixed), the coefficient $\tan (\theta) / r$ is a constant. Thus, the tangential component of the particle acceleration and the sound pressure oscillate synchronously. Therefore, at the instant of a pressure null, the tangential component of the particle acceleration $\left(-1 /\left(\rho_{0} r\right) \tan (\theta) p(r, \theta, t ; \omega) \mathbf{e}_{\theta}\right)$ vanishes, whereas at the same instant the radial component $\left.((1+1 /(1+j k r)) / r+j k) / \rho_{0} p(r, \theta, t ; \omega) \mathbf{e}_{r}\right)$ is non-vanishing due to the phase difference resulting from the imaginary term ' $j k+(r(1+j k r))^{-1}$ '. Of course, this conclusion does not apply to the transverse plane $\left(\theta=90^{\circ}\right)$ where sound pressure vanishes altogether. It is easily verified that for the emission of sound of arbitrary waveform by the dipole (where we must add over a series of frequencies) the same result holds, because the coefficient $\tan (\theta) / r$ is independent of the frequency. Thus, at distances large compared with the displacements of the oscillating sphere, the particle acceleration is directed towards or away from the source at the instant of a pressure null. From inspection of (2) we observe that the particle acceleration leads the sound pressure. Thus, $\mathbf{a}(r, \theta, t ; \omega)$ points to the source at the instant of a negative zero-crossing of the sound pressure. Basically, the above result is a consequence of the separability of the expression for the sound pressure of a dipole in spherical coordinates:

$p(r, \theta, t ; \omega)=A(r) B(\theta) \exp (j(\omega t-k r)$

with $\mathrm{A}$ a complex function of ' $r$ ' and $\mathrm{B}$ a real function of $\theta$. Hence,

$$
\begin{aligned}
\mathbf{a}(r, \theta, t ; \omega)= & -1 / \rho_{0}\left[\mathrm{~B}^{\prime}(\theta) /(r \mathbf{B}(\theta)) p\left(r, \theta, t_{j} \omega\right) \mathbf{e}_{\theta}\right. \\
& \left.+\left(\mathrm{A}^{\prime}(r)-j k\right) / \mathbf{A}(r) p(r, \theta, t ; \omega) \mathbf{e}_{r}\right]
\end{aligned}
$$

After these preliminaries the generalization to arbitrary sound sources is straightforward. The nature of our very question suggests an analysis of solutions in spherical coordinates because such solutions make explicit the radial and the tangential components of the particle motion. For sources with small dimensions and small excursions with respect to the wavelength of the sound and axial symmetry of the radiation pattern, the expression for the sound pressure may be found in Harris (1964). The general solution not restricted to axial symmetry may be found in Lamb (art. 297, p. 505) but will not be used here. The arguments put forward in 
the next section, however, pertain to this more general case also.

$$
\begin{aligned}
& p(r, \theta, t)=\sum_{\omega} p(r, \theta, t ; \omega) \\
& p(r, \theta, t ; \omega)=\sum_{m=0}^{\infty} A_{m}\left[J_{m}(k r)+j N_{m}(k r)\right] \\
& \times P_{m}[\cos (\theta)] \exp (j \omega t)
\end{aligned}
$$

Each term $m$ in the series describes the contribution of a source of increasing complexity to the field. The first three terms represent a radially pulsating source, a translatory oscillation of a spherical source and a deforming source, respectively (monopole, $m=0$; dipole, $m=1$; quadrupole, $m=2$ ). The field of a source of arbitrary complexity may be described by taking sufficiently many terms of the series into account. However, as the contributions specific to the higher order terms $(m>0)$ decrease with increasing power over distance, the field is in practice determined by the lowest order non-zero source term except very near the source. Notice that (5) is separated with respect to spherical variables $(r, \theta)$. Thus, variation of the distance $(r)$ or the angle $(\theta)$ have independent effects on the local sound pressure. The exact form of the solution is not important to our argument and will not be given here. Readers may find the mathematical details in the Appendix.

The Legendre polynomials of degree $m\left(P_{m}\right)$ that describe the angular dependence of the radiation pattern are real and independent of the sound frequency. Thus, variation of the angular position (and keeping $r$ constant) results in a simple change of the amplitude of the local sound pressure, which is independent of the spectral content of the signal. On the other hand, the distancedependent part of the solution [represented by the Bessel $\left(J_{m}\right)$ and Neuman $\left(N_{m}\right)$ functions] contains an imaginary term also. This complex part of the solution provides the phase relationship between different locations in the field. Apparently, the phase relation between the sound pressures at two different locations in the field varies only as a function of the wavelength-scaled distance $(k r)$. Thus, the local pressure variations are in phase or antiphase on spherical surfaces around the source irrespective of the order $m$, and the sound pressure nulls are coincident in time on spherical surfaces around the source. This means that the pressure gradient along the spherical surface vanishes at the instant of the pressure null and therefore also the particle acceleration tangential to this surface [cf. $\mathbf{e}_{\boldsymbol{\theta}}$ part of (2)]. Again, adding over an infinite series of frequencies, the result may be generalized to arbitrary waveforms. Thus, we may conclude that for sources of arbitrary order (with axial symmetry) the particle acceleration is radially directed at the instant of the zero-crossing of the sound pressure. It appears then that a strategy in which the particle acceleration is sampled at the instants of pressure nulls would in principle allow the fish to recover the direction towards the source with a $180^{\circ}$ ambiguity remaining. This ambiguity could be solved by analysis of the temporal relation between $p$ and $a_{r}$.
There are a number of limitations of the proposed strategy, however. Firstly, the strategy only holds for any region where the sound pressure is non-vanishing. Secondly, when the temporal phase difference between the radial and the tangential acceleration components is small, the radial component nearly vanishes at the instant of the pressure null and may become difficult to detect. This is the case for locations in the vicinity of the source where the particle acceleration is dominated by the (irrotational) flow. Thirdly, pressure noise will shift the instants of the pressure null. This leads to sampling at instants where the particle acceleration is not exactly radially directed.

Simulations of the sound field in the vicinity of a quadrupole to be discussed below will show that the proposed mechanism is rather robust against pressure noise.

\section{Distance information}

There is some evidence that fish may be able to perceive the distance of the sound source. Schuijf and Hawkins (1983) showed discrimination of sound sources placed at different distances by codfish. Here we do not wish to speculate on the perceptual abilities of fish in this respect but merely consider the question whether there is local distance information present in the soundfield.

First, there is no unambiguous distance information present in the amplitude or spectral content of any of the local sound field variables in isolation, because any variation of these variables with distance may be compensated by an appropriate change of the source strength or spectral content of the sound emission.

Secondly, we may remark that the relation (be it with respect to amplitude, to temporal or to a combination of both) between the tangential component of the particle acceleration and the sound pressure cannot provide distance information, because it depends on $\tau$ and $\theta$. It seems appropriate then to limit our attention to the relation between sound pressure and radial particle acceleration.

By partial differentiation of $p$ to the distance, we may obtain the expression for the radial particle acceleration $\left(a_{r}\right)$ at a specific frequency $\omega$.

$a_{r}(r, \theta, t ; \omega)=-1 / \rho_{0}\left(\nabla p \cdot \mathbf{e}_{r}\right)=-1 / \rho_{0} \delta p / \delta r$

$p(r, \theta, t ; \omega)=A(k r) B(\theta) \exp (j \omega t)$

Recall that the sound pressure is separated with respect to its angular and radial parts and that its dependence on the angular components is real. Partial differentiation of the complex function $A(k r)\left(=J_{m}(k r)+j N_{m}(k r)\right)$ is equivalent to multiplication by a complex number specific to each distance and frequency

$\delta A(k r) / \delta r=Z_{r}(k r ; \omega) A(k r)$

Comparison of the expressions for the radial component of the particle acceleration and the sound pressure (6) 
shows that for each frequency $(\omega)$ their relation is fully captured by the function $Z_{r}$. Unlike the sound pressure and the radial particle acceleration, the function $Z_{r}$ is no longer dependent on the angular variable ' $\theta$ ', but only varies with wavelength-scaled distance $(k r)$ for the frequency considered

$a_{r} / p=Z_{r}(k r ; \omega)$

Notice, however that the frequency parameter ' $\omega$ ' and the variable ' $k r$ ' are related as

$k r=2 \pi r / \lambda=\omega r / c$

where $c$ denotes the speed of sound in water. Thus, $Z_{r}(k r ; \omega)$ may also be analysed as $Z_{r}(\omega ; r / c)$ which means that the relation between $a_{r}$ and $p$ conforms to a spectral filtering in a distance-dependent way. The exact form of the filter is also dependent on the source type. Expressions for $Z_{r}(k r ; \omega)$ may be found in the Appendix. Fig. 1 shows the shape of $Z_{r}(\omega ; r / c)$ for $r / c=0.001,0.01$ and 0.1 (corresponding to distances of $1.5,15$ and $150 \mathrm{~m}$, respectively, in seawater) for three different source types (mono, di- and quadrupole). At all distances the $a_{r} / p$ ratio is nearly constant and practically in phase up to a critical frequency. Above the critical frequency, this ratio increases proportionally to the frequency, whereas the phase shift between $a_{r}$ and $p$ rapidly approaches $90^{\circ}$. The critical frequency may be defined as the frequency where $a_{r}$ leads $p$ by $45^{\circ}$. From Fig. 1 we may observe that the critical frequency equals about $2 \mathrm{~Hz}, 20 \mathrm{~Hz}$ and $200 \mathrm{~Hz}$ for distances of 150,15 and $1.5 \mathrm{~m}$, respectivey. The variation of the critical frequency for different source types is of the order of an octave. Thus, if the fish perceives both the sound pressure and the particle acceleration component of the sound, it may in principle derive absolute distance estimates from the analysis of the spectral relation between those components.

The relation between the tangential particle acceleration and the sound pressure may similarly be captured by a complex number $\left(Z_{\theta}\right)$, which is now only dependent on the source distance and the angle $\theta$. By partial differentiation of $p$ to $\theta$ we obtain the expression for the tangential particle acceleration $\left(a_{\theta}\right)$ at a specific distance $r$.

$$
\begin{aligned}
a_{\theta}(r, \theta, t ; \omega) & =-1 /\left(\rho_{0} r\right)\left(\nabla p \cdot \mathbf{e}_{\theta}\right) \\
& =-1 /\left(\rho_{0} r\right) \delta p / \delta \theta \\
p(r, \theta, t ; \omega) & =A(k r) P_{m}[\cos (\theta)] \exp (j \omega t)
\end{aligned}
$$

$P_{m}[\cos (\theta)]$ stands for the angular part of the solution of the wave equation for axially symmetric sound sources of order $m$. Using the relation (Abramowitz and Stegun 1970)

$$
\begin{aligned}
\frac{\delta P_{m}[\cos (\theta)]}{\delta \theta}= & \frac{-(m+1)}{\sin (\theta)}\left[\cos (\theta) P_{m}(\cos (\theta)\right. \\
& -P_{m+1}(\cos (\theta)]
\end{aligned}
$$

we obtain for $Z_{\theta}=a_{\theta} / p$,

$Z_{\theta}=\frac{m+1}{\rho_{0} r \sin (\theta)}\left\{\cos (\theta)-\frac{P_{m+1}[\cos (\theta)]}{P_{m}[\cos (\theta)]}\right\}$
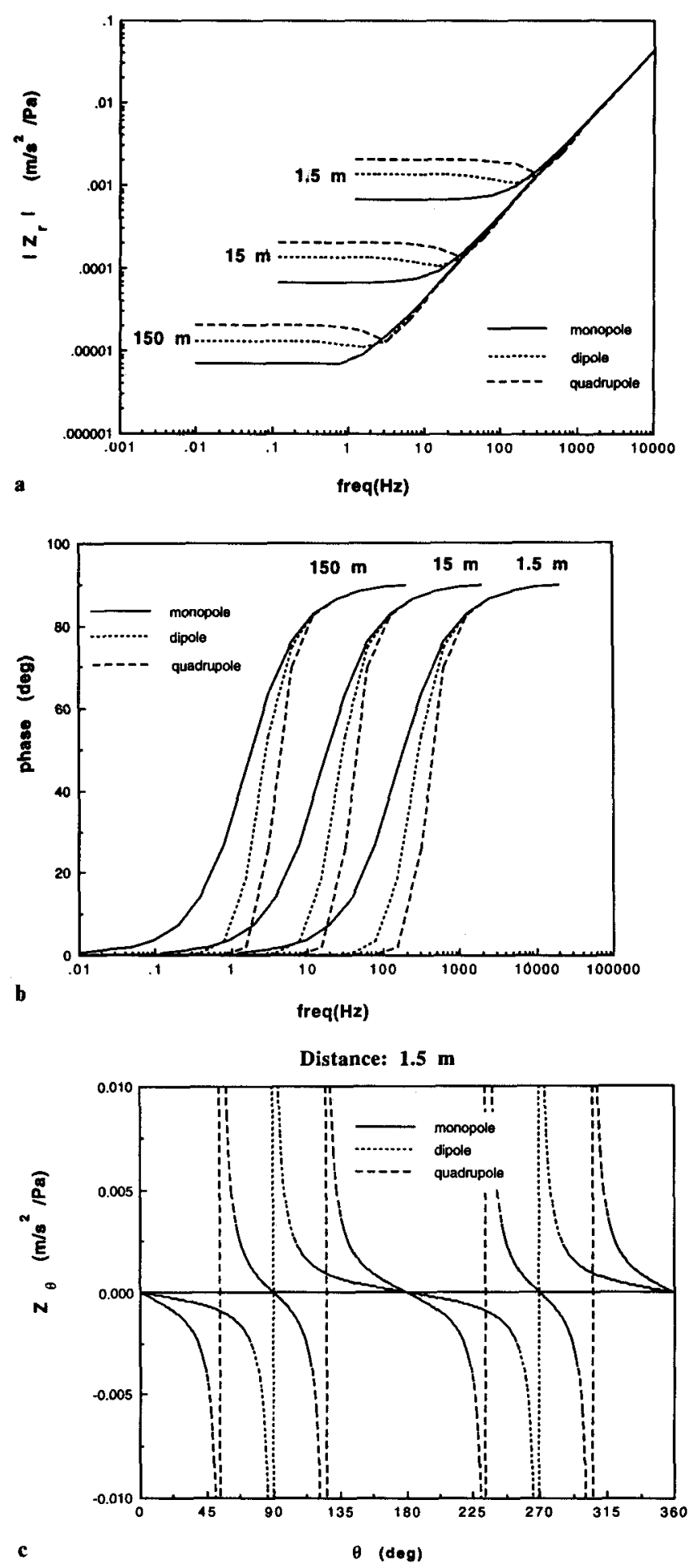

Fig. 1a-c. Characteristics of the three lowest order sound sources with axial symmetry under free-field conditions Amplitude ratio $\left(\left|Z_{r}\right|\right)$ and phase angle of the relation between radial particle acceleration and sound pressure are shown in a and b, respectively. Indicated are spectral relations for monopole, dipole and quadrupole fields, for three different distances $(1.5 \mathrm{~m}, 15 \mathrm{~m}$ and $150 \mathrm{~m})$. c The amplitude ratio $\left(Z_{\theta}\right)$ of the tangential particle acceleration and the sound pressure at a distance of $1.5 \mathrm{~m}$ as a function of the direction $(\theta)$ relative to the symmetry axis of the source. The latter amplitude ratio is inversely proportional to the distance 
Figure 1C shows $Z_{\theta}$ for the same source types as were used for the computations of $Z_{r}(k r ; \omega)$ for a distance to the source of $1.5 \mathrm{~m}$. Combining Fig. $1 \mathrm{~A}$ and $\mathrm{C}$ we may derive the amplitude ratio between the tangential and the radial particle acceleration for any angle $\theta$ at a distance of $1.5 \mathrm{~m}$. Because $Z_{\theta}$ is inversely proportional to the distance $(r)$, and $Z_{r}$ shifts left downward along the highfrequency asymptote when the distance is increased, values for other distances can also be derived with the aid of these figures. The phase difference between $a_{r}$ and $a_{\theta}$ equals that indicated in Fig. 1B with the proviso that for negative values of $Z_{\theta}$ a $180^{\circ}$ phase shift should be added.

Using the expressions for $Z_{r}(k r ; \omega)$ and $Z_{\theta}$ we may now specify more accurately the limits on the mechanism of directional hearing by sampling of the particle acceleration on the instants of the pressure nulls. Because the particle acceleration has no tangential components for a monopole, we concentrate on the properties of the dipole and quadrupole fields.

The singular points in Fig. 1C arise at locations where the sound pressure vanishes. At these points the mechanism fails because the reference signal for sampling is absent. For the dipole this occurs at $\theta=90^{\circ}$ and $\theta=270^{\circ}$. Because the source is assumed to be axially symmetrical, this means that the singularities are found in the plane perpendicular to the dipole axis and halfway between the poles of the acoustic doublet. For the quadrupole the singular points in Fig. $1 \mathrm{C}$ are found at $\theta=54.7^{\circ}\left(305.3^{\circ}\right)$ and $\theta=125.3^{\circ}\left(234.7^{\circ}\right)$, approximately. Thus, the singular points lie in conical surfaces with their axis parallel to the symmetry axis of the source.

Depending on the pressure noise level, the reference signal may not be detectable for a larger range of elevations around the critical elevation where the sound pressure vanishes.

The temporal relation between the radial particle acceleration and the sound pressure puts a second restriction on the mechanism. The mechanism relies on the vanishing of the tangential particle acceleration at the instants of the pressure nulls. Obviously, no directional information is gained when the radial particle acceleration vanishes also at the instants of the pressure nulls. This is practically the case when the frequency content of the signal is restricted to the range below about half the critical frequency. For these low frequencies the phase advance of $a_{r}$ relative to $p$ is less than $5^{\circ}$ for the dipole and the quadrupole (Fig. 1B). Thus, at the instant of the pressure null both the tangential and the radial component of the particle acceleration vanish.

\section{Simulation of the quadrupole field}

To investigate the robustness of the sampling mechanism for directional hearing, we computed the particle acceleration at $1-\mathrm{ms}$ intervals for a total period of $1 \mathrm{~s}$. Four different angular locations $\left(\theta=10^{\circ}, 30^{\circ}, 50^{\circ}\right.$ and $\left.70^{\circ}\right)$ and two different distances $(2.5$ and $10 \mathrm{~m})$ with respect to the source were investigated. The simulations were done in one quadrant because of the mirror symmetry of the quadrupole field with respect to the $\theta=0^{\circ}$ and $90^{\circ}$ axes.
The local sound pressure signal was a narrow band noise (gaussian, centre frequency $200 \mathrm{~Hz}, \mathrm{SD}$ $22.5 \mathrm{~Hz}$ ) with unit magnitude (see inset of Fig. 2). Using the above expressions for $Z_{\mathrm{r}}$ and $Z_{\theta}$, the radial and the tangential particle acceleration were computed. The set of particle acceleration vectors thus obtained were plotted in a cartesian reference frame (units: $p Z_{r} * 1000$ and $p Z_{\theta} * 1000$ ).

As shown in Fig. 2, the particle acceleration vectors form a broad distribution of directions.

The major axis is nearly radially directed at $10 \mathrm{~m}$ distance except at $\theta=50^{\circ}$. For the latter position as well as for all positions at $2.5 \mathrm{~m}$ distance, the particle acceleration has a strong tangential component for all locations. The major axes of the latter particle acceleration distributions deviate by $20-80^{\circ}$ from the radial direction.

The direction of the particle acceleration at the instants of the pressure nulls was computed in the presence of broadband pressure noise. The pressure noise was also gaussian (centre frequency $200 \mathrm{~Hz}, \mathrm{SD} 225 \mathrm{~Hz}$ ). The total-power $\mathrm{S} / \mathrm{N}$ ratio (SNR) was $20 \mathrm{~dB}$ or $40 \mathrm{~dB}$. Linear interpolation of the particle acceleration vector was done when the pressure null was located between two successive sound pressure samples. The sampled particle acceleration vectors were grouped in bins with a width of $20^{\circ}$. This grouping was motivated by the fact that the discrimination angle of sound source direction by fish typically is of the order of $20^{\circ}$.

Figures 3 and 4 show direction characteristics for each location. For each bin, a count was made of the number of sound pressure nulls where the concurrent particle acceleration vector was directed within the range of the bin. This count is shown in a polar plot against the direction of the bin. In the figures, each direction characteristic is rotated in order to align the bins which contain the radial particle acceleration count with the radius towards the source for each location. Clearly, when the SNR is $40 \mathrm{~dB}$, practically all the particle acceleration vectors are radially directed, irrespective of the location. Only at a distance of $2.5 \mathrm{~m}$ at $\theta=50^{\circ}$ was a significant fraction of the sampled acceleration vectors not radially directed. Nevertheless, the major axis of this 'direction characteristic' was radially directed, indicating that the mechanism may substantially improve direction detection when the tangential particle acceleration far outweighs the radial acceleration component. Comparing the major axis direction of the raw particle acceleration plots (Fig. 2) and the directions of the sampled acceleration vectors (Fig. 3), it is clear that the sampling mechanism potentially provides a powerful contribution to the detection of the source direction in the fields of higher order sound sources.

Similar conclusions apply to the majority of the simulations with a SNR of $20 \mathrm{~dB}$. In general, the 'direction characteristics' are broader, but the major axis is close to radially directed and deviates less than $10^{\circ}$. The notable exception is the polar plot at $2.5 \mathrm{~m}$ distance and $\theta=50^{\circ}$. In this case, the mechanism clearly fails to recover the source direction. This is due to the large ratio between the tangential and the radial particle acceleration $(>5)$, in combination with the temporal jitter of the pressure 


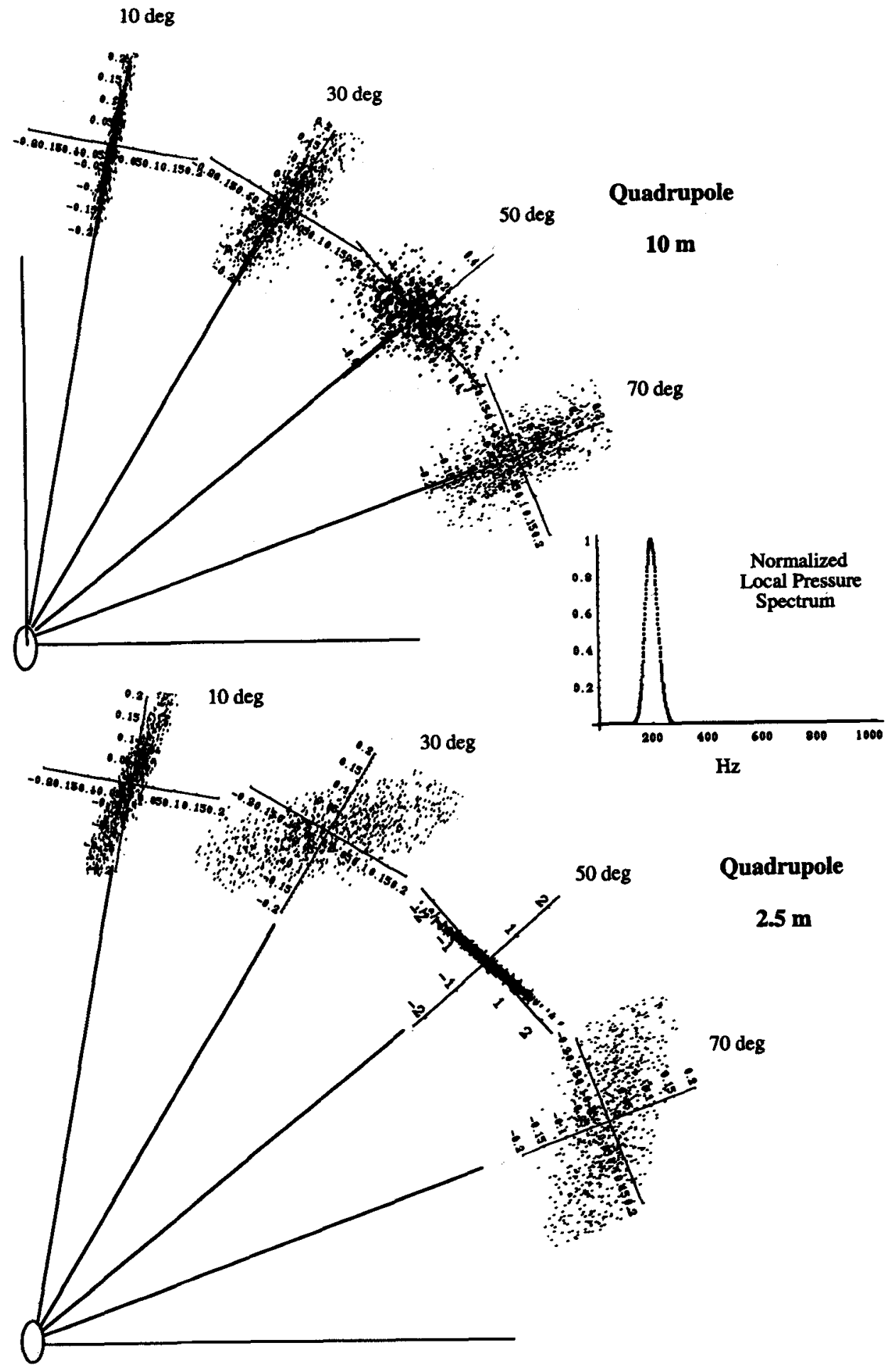

Fig. 2. Local particle acceleration vectors were computed in the soundfield of a quadrupole at four different locations concentric with respect to the source $\left(\theta=10^{\circ}, 30^{\circ}, 50^{\circ}\right.$ or $\left.70^{\circ}\right)$ and at two different distances (upper panel $10 \mathrm{~m}$; lower panel $2.5 \mathrm{~m}$ ). Each plot at each location contains 1000 particle acceleration vectors. For clarity, the plots are rotated so as to direct the radial component of the particle acceleration towards the source, which is indicated by the ellipse in the lower left corner of each panel. Particle acceleration is computed with 1-ms intervals assuming for the local sound pressure spectrum the characteristic illustrated in the inset (gaussian; centre frequency $200 \mathrm{~Hz}$; SD $22.5 \mathrm{~Hz}$ ). When the arbitrary unit of the sound pressure is chosen such that the spectrum peak is $1 \mathrm{~Pa} / \mathrm{Hz}$, then the unit of the particle acceleration indicated by the figures is $1 \mathrm{~mm} / \mathrm{s}^{2}$

nulls due to the pressure noise. Another reason is the small phase difference between the tangential and the radial particle acceleration at $200 \mathrm{~Hz}$ for this distance. Consequently, $a_{r}$ nearly vanishes at the instants of the pressure nulls. This amplifies the effect of the temporal jitter of the pressure nulls on the direction of the sampled acceleration vector.

\section{Discussion}

We have analyzed the information available to a fish for the solution of the sound source localization problem. As mentioned before, the scope of the analysis is restricted in a number of respects. First, our results apply to an unbounded medium. The contributions of reflections to 


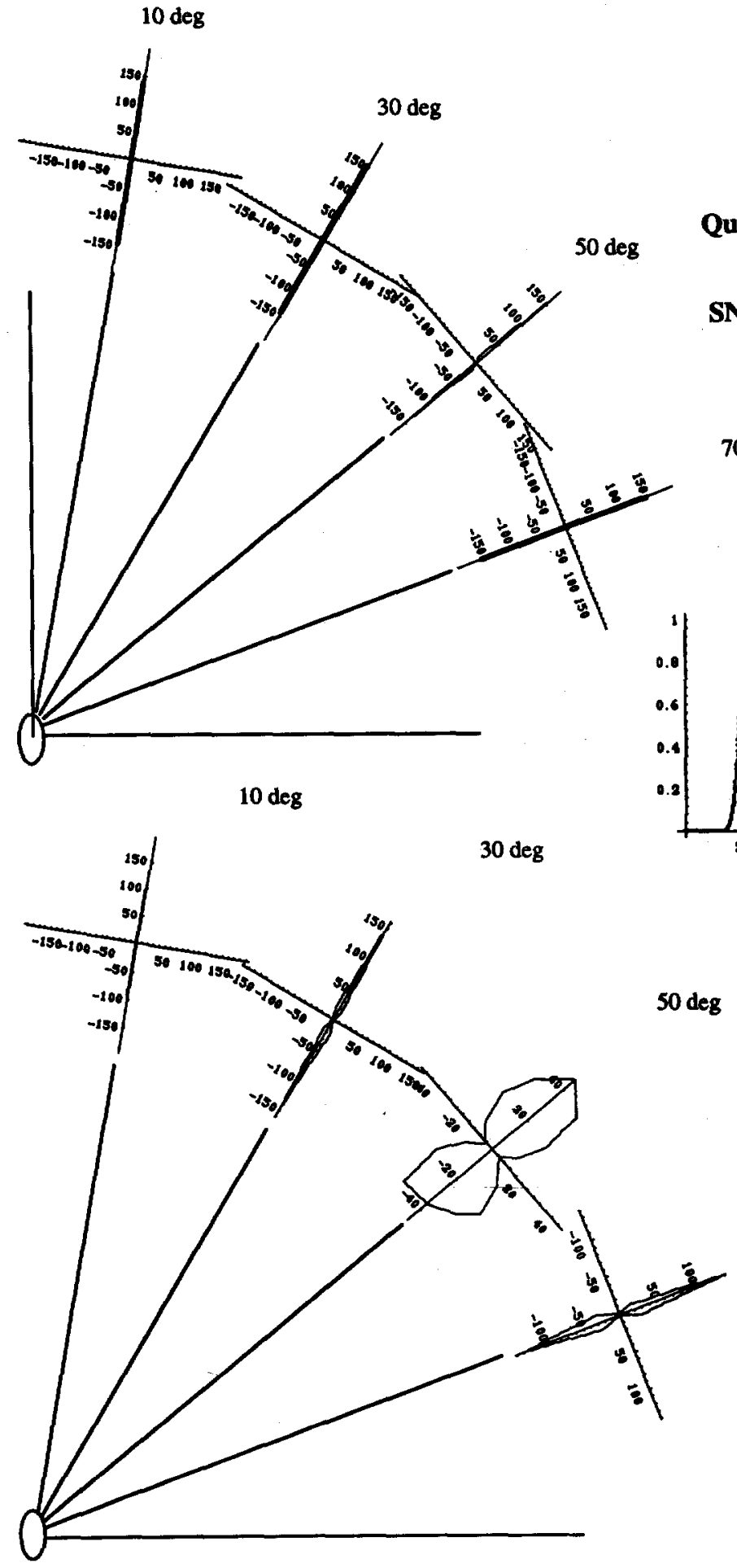

\section{Quadrupole}

SNR: $40 \mathrm{~dB}$

$70 \mathrm{deg}$

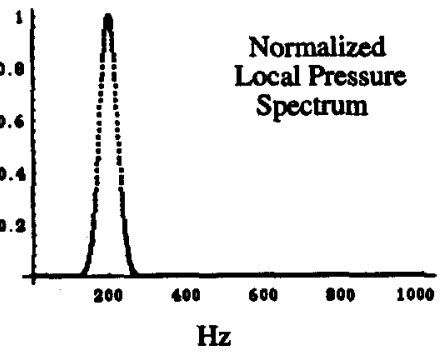

the water surface and the bottom are neglected. Thus, the analysis is valid for source localization in deep and extended water basins such as the sea and large lakes.

Secondly, we have analyzed the problem for 'on the spot' source localization. This is - admittedly - a more difficult task than when the fish is free to make exploratory movements and can sample the field over an

\section{Quadrupole $2.5 \mathrm{~m}$}

SNR: $40 \mathrm{~dB}$

$70 \mathrm{deg}$

extended spatial and temporal range. This might raise the question whether this analysis is not merely an academic matter. We feel, however, that this is not the case. As mentioned before, there is evidence that fish are able to localize a sound source nearly instantaneously (for a more detailed discussion of these abilities see Shuijf and Buwalda 1980; Buwalda 1981; and Shuijf 1981). 


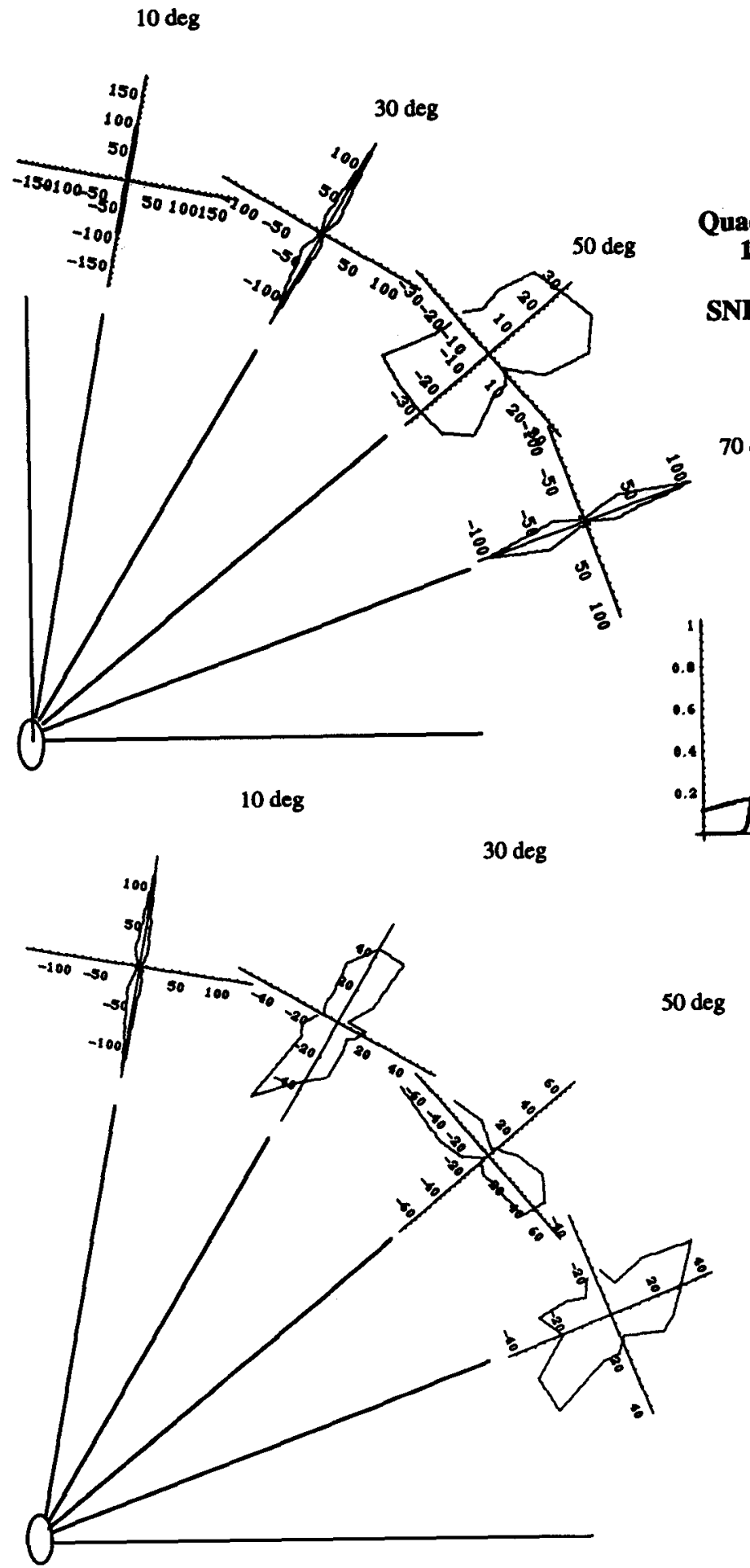

Fig. 4. Direction characteristics of the sampling mechanism at a signalto-noise ratio of $20 \mathrm{~dB}$. Other simulation conditions as described in the legend of Fig. 3

Moreover, sound emitted by natural sources is usually discontinuous (e.g. sound of struggling fish, of diving birds feeding on a school of fish, of stridulation or of swimbladder pulsations). Source localization based on gradient search is complicated for such conditions by the irregular modulation of the sound and may be impossible for brief sounds emitted with long intervals. Clearly, the capability of 'instantaneous' localization is then advantageous.

We have shown that at the instant of a pressure null the particle acceleration is radially directed for sources of any order. Moreover, the distance to the source is implied in the spectral relation between the radial particle acceleration and the sound pressure in a source-dependent way 
(see Fig. 1). Thus, in principle the source localization problem can be solved fully, i.e. with respect to source direction and source distance, by a local reading of the soundfield. In addition, information can be gained about the order of the source. Apparently, even some source identification can be done based on the analysis of the local relation between $p$ and $a_{r}$. Notice that this type of source identification is not dependent on the exact waveform of the sound emission provided that the bandwidth is sufficiently large.

Although the simulations showed that the particle acceleration is not always exactly radially directed at the sampling instants because of temporal jitter by pressure noise, the mechanism may nevertheless be beneficial for source localization in the sense that sampling narrows the set of perceived directions to a range much closer to the true source direction compared with the full set of local particle acceleration directions.

For higher order sources, particle motion may occur along a three-dimensional trajectory, which implies that the direction of the source as indicated by the particle motion component by itself can be ambiguous on the sphere! Our analysis shows that for conditions under which higher order source terms contribute significantly to the field, the fish need not necessarily rely on the lateral line or some sort of gradient search for sound source localization, as has been suggested previously (Kalmijn 1988). In principle, a local reading of the sound field provides sufficient information to solve the source localization problem. It appears that for directional hearing, the principle of time-sampling of the particle acceleration direction based on the zero-crossings of a coherent reference wave $(p)$ is essential to overcome ambiguities due to non-radial particle motion components in the field of the external sound source. Interestingly, this very principle was formulated by Schuijf (1981) as a means to disambiguate the particle motion direction of the external field from that due to the scatter field of the swimbladder.

Currently, little evidence exists concerning source localization abilities of fish towards sound sources with a higher order of complexity than monopoles. Thus, we can only speculate to what extent the cues we have made explicit are really used by the fish. We know though that the cod is able to analyze the direction of the particle motion depending on the phase of the sound pressure in a task where $180^{\circ}$ ambiguity exists concerning the source direction. Thus, in a weak form this fish has been shown to possess the required ability of timing analysis. In addition, Van den Berg (1985) showed that the cod can discriminate the direction of revolution of elliptical particle motion trajectories when the minor to major axis ratio exceeded $-12 \mathrm{~dB}$. Apparently, this fish is able to process the minor directional component in the presence of a much larger perpendicularly oriented component. This relative absence of masking between perpendicularly oriented motion components would seem a prerequisite to any mechanism that seeks to enhance the possibly weak radial component with respect to the tangential component of the particle acceleration.
We would like to conclude our analysis with a tentative functional description of a possible sound source localization system based on the above cues. The hypothetical system consists of one non-directional soundpressure-sensitive channel and a set of particle-acceleration-sensitive channels. The channels are supposed to possess negligible cross-modality sensitivity. Buwalda (1981) has shown that this condition is met approximately in the cod. The preferred directions of the directional channels are evenly distributed over the sphere. The acceleration channels are combined in pairs with perpendicular directional preferences. Such a pair defines a plane on the sphere of possible source directions. At the instant of a pressure null, the particle acceleration is radially directed. Thus, at this instant the channels of pair with sensitivity axes in a plane perpendicular to the radial particle acceleration make a synchronous zero crossing. Conversely, the synchronous zero crossings of such a channel pair with the zero crossing of the $p$ channel identify the direction of the source up to a $180^{\circ}$ ambiguity. The acceleration channel with preferred sensitivity perpendicular to those of the aforementioned pair is directed along the radius through the source and the fish. The zero crossings of the latter channel are coincident with the zero crossings of the radial particle acceleration. Thus, the system could be organized as a set of orthogonal triads of particle-acceleration-sensitive channels. The temporal analysis between the two channels of the triad which make synchronous zero crossings (coincident with zeroes of $p$ ) and the zero crossings of the third channel may provide a rough estimate of the distance and the order of the source. This can for instance be realized by a set of parallel temporal filters described by the functions $Z(\omega ; r / c)$, each corresponding to a particular combination of source type (mono-, dipole, etc.) and distance $(r / c)$. For a rough classification of the distance between the nose and infinity in, say five categories and five different source types, only 25 filters would be necessary. Parallel filtering of the signal of the $p$-channel (or of the channel pair with the synchronous zero crossings) provides the fish with a set of 'expected' radial particle acceleration waveforms based on the hypotheses that the source is located at the distance and is of the order as specified by the filters. The task for the fish to classify the order of the source and its distance then boils down to finding the 'expected' radial particle acceleration which best matches the signal in the radial particle acceleration channel. In this scheme the analysis is based on the temporal sequence of the zero crossings in the $p$-channel and the radially directed particle acceleration channel. At first sight, this might seem problematical because a lot of information appears to be neglected. This is not the case though. A similar analysis stressing the importance of zero crossings was put forward by Marr (1982) for the analysis of images by the visual system. In this twodimensional case, severe problems may arise, but for one-dimensional signals, a formal proof has been given that the sequence of zero crossings fully describes the waveform up to a scale transformation (Logan's theorem). Thus, for a temporal analysis as proposed in the above model, accurate coding of the zero crossings of the 
acoustical signals is sufficient. On the other hand, there is a reason to stress the importance of temporal over amplitude coding. The pressure-induced swimbladder pulsations are dependent on its compliance. At greater depth, the stiffness of the swimbladder increases, due to the larger hydrostatic pressure, resulting in induced oscillations of smaller amplitude. Hence, the amplitude encoded by the $p$-channel is depth dependent. Consequently, the $a_{r}-p$ amplitude ratio cannot be encoded by the fish in a depth-independent way, resulting in ambiguities in the distance estimate if based on this amplitude ratio.

\section{Appendix}

The solution to the wave equation in spherical coordinates may be found in Lamb (1931; art. 197, p. 505). The velocity potential ' $\Phi$ ' is specified for any location in the field of a source of general complexity by a doubly infinite series:

$\Phi=\sum_{\omega} \sum_{n} \Phi_{n}(r, \theta, \phi, t ; \omega)$

where ' $\Phi_{n}$ ' denotes the contribution of a source term of order ' $n$ ' to the field at circular frequency ' $\omega$ '

$\Phi_{n}(r, \theta, \phi, t ; \omega)=A_{n} \zeta^{n} f_{n}(\zeta) \frac{S_{n}(\theta, \phi)}{k^{n}} \exp (j \omega t)$

where $j=(-1)^{1 / 2}$

$\omega=2 \pi f$

$\zeta=k r$

$k=2 \pi / \lambda$

$r=$ distance in meters

$\lambda=$ wavelength in meters

$t=$ time in seconds

$f=$ frequency in $\mathrm{s}^{-1}$

$c=$ speed of sound in water $(\mathrm{m} / \mathrm{s})$

The functions $\zeta^{n} f_{n}(\zeta)$ and $S_{n}(\theta, \phi)$ specify the radially dependent part and the spherical dependence of ' $\Phi_{n}$ ', respectively. The spherical harmonics of order ' $n$ ' are real and given by:

$$
\begin{aligned}
S_{n}(\theta, \phi)= & B_{0} P_{n}[\cos (\theta)] \\
& +\sum_{l=1}^{n}\left(B_{l} \cos (l \phi)+D_{l} \sin [(l \phi)] P_{n}^{l} \cos (\theta)(A 3)\right.
\end{aligned}
$$

with $B_{l}$ and $D_{l}$ arbitrary constants.

As we are mainly interested in the distance-dependent part of the solution, the explicit expressions for the Legendre polynomials ' $P_{n}^{l} \cos (\theta)$ ' of degree ' $n$ ' and order ' $l$ ' are not reproduced here. These may be found in Lamb (1931; art. 86, p. 117). The distance-dependent part specifies the solution for expanding waves.

$$
\begin{aligned}
& \zeta^{n} f_{n}(\zeta)=\frac{j^{n} \exp (-j \zeta)}{\zeta}\left[\sum_{l=0}^{n} g_{l}^{n}\right] \text { and } \\
& g_{l}^{n}=\frac{\prod_{i=1-1}^{l}(n+i)}{\prod_{i=1}^{l}(j 2 i \zeta)}, l>0 ; \quad g_{0}^{n}=1
\end{aligned}
$$

We remark that this function is complex and consists of distance-dependent real and imaginary parts. It is now a straightforward matter to derive the expressions for the radial particle acceleration and the sound pressure,

$$
\begin{aligned}
p_{n}(r, \theta, \phi, t ; \omega) & =-\rho_{0} \frac{\delta \Phi_{n}}{\delta t} \\
& =-\rho_{0} \mathrm{c} A_{n} \zeta^{n} f_{n}(\zeta) \frac{S_{n}(\theta, \phi)}{k^{n-1}} \exp (j \omega t)
\end{aligned}
$$

and

$$
\begin{aligned}
a_{r, n}(r, \theta, \phi, t ; \omega) & =-1 / \rho_{0}\left(\nabla p_{n} \cdot \mathbf{e}_{r}\right)=-k / \rho_{0} \frac{\delta p_{n}}{\delta \zeta} \\
& =j k c A_{n}\left(n \zeta^{n-1} f_{n}(\zeta)\right. \\
& \left.-\zeta^{n+1} f_{n+1}(\zeta)\right) \frac{S_{n}(\theta, \phi)}{k^{n-1}} \exp (j \omega t)
\end{aligned}
$$

Equations (A5) and (A6) were used to derive the $Z_{r, n}=a_{r, n} / p_{n}$ ratios. $S_{n}(\theta, \phi)$ was not evaluated in these computations because the $a_{r, n} / p_{n}$ ratio is independent of the spherical variables. The complex function $Z_{r, n}$ was evaluated for $n=0$ (monopole), $n=1$ (dipole) and $n=2$ (quadrupole). Subsequently, the $Z_{r, n}$ curves as a function of the wavelength-scaled distance were transformed with the following identity to yield the $a_{r} / p$ ratio as a function of the sound frequency:

$Z(k r)=Z(\omega ; r / c)$

taking $r / c$ at $0.001,0.01$ and 0.1 , i.e. at distances of $1.5,15$ and $150 \mathrm{~m}$ from the source, respectively.

\section{References}

Abramowitz M, Stegun A (1970) Handbook of mathematical functions, 9 th edn. Dover Press, New York, p 344

Berg AV van den (1985) Analysis of the phase difference between particle motion components of sound by teleosts. J Exp Biol 119:183-197

Buwalda RJA (1981) Segregation of directional and nondirectional acoustic information in the cod. In: Tavolga, WN, Popper AN, Fay RR (eds) Hearing and sound communication in fishes. Springer, Berlin Heidelberg New York, pp 139-171

Buwalda RJA, Hawkins AD, Schuijf A (1983) Discrimination by the cod of sounds from opposing directions. J Comp Physiol 150:175-184

Chapman CJ, Johnstone ADF (1974) Some auditory discrimination experiments on marine fish. J Exp Biol 61:521-528

Harris GG (1964) Considerations on the physics of sound production by fishes. In: Tavolga WN (ed). Marine bio-acoustics. Pergamon Press, Oxford, pp 233-247

Hawkins AD, Sand O (1977) Directional hearing in the median vertical plane by the cod. J Comp Physiol 122:1-8

Kalmijn AJ (1988) Hydrodynamic and acoustic field detection. In: Atema J, Fay RR, Popper AN, Tavolga WN (eds). Sensory biology of aquatic animals. Springer, Berlin Heidelberg New York, pp. $83-130$

Kinsler LE, Frey AR, Coppens AB, Sanders JV (1982) Fundamentals of acoustics, 3rd edn. Wiley, New York

Lamb H (1931) Hydrodynamics, 6th edn. Cambridge University Press, London 
Logan BF (1977) Information in the zero-crossings of bandpass signals. Bell Syst Tech J 56:487-510

Maniwa Y (1976) Attraction of bony fish, squid and crab by sound. In: Shuijf A, Hawkins AD (eds). Sound reception in fish. Elsevier, Amsterdam, pp 271-282

Marr D (1982) Vision. W.H. Freeman, San Francisco

Myrberg AA Jr, Ha SJ, Walewski S, Banbury JC (1972) Effectiveness of acoustic signals in attracting epipelagic sharks to an underwater sound source. Bull Mar Sci 22:926-949

Nelson D (1965) Hearing and acoustic orientation in the lemon shark Negaprion brevirostris (poey) and other large sharks. $\mathrm{PhD}$ thesis, University of Miami

Nelson D, Johnson R (1972) Acoustic attraction of Pacific reef sharks: effect of pulse intermittency and variability. Comp Biochem Physiol 42:85-95

Oslen K (1976) Evidence for localization of sound by fish in schools. In: Shuijf A, Hawkins AD (eds). Sound reception in fish. Elsevier, Amsterdam, pp 257-268

Richard JD (1968) Fish attraction with pulsed, low frequency sound. J Fish Res Bd Can 25:1441-1452
Sand O (1981) The lateral line and sound reception. In: Tavolga WN, Popper AN, Fay RR (eds). Hearing and sound communication in fishes. Springer, Berlin Heidelberg New York, pp 459-478

Shuijf A (1976) The phase model of directional hearing in fish. In: Shuijf A, Hawkins AD (eds). Sound reception in fish. Elsevier, Amsterdam, pp 63-88

Schuijf A (1981) Models of acoustic localization. In: Tavolga WN, Popper AN, Fay RR (eds). Hearing and sound communication in fishes. Springer, Berlin Heidelberg New York, pp 267-310

Schuijf A, Buwalda RJA (1975) On the mechanism of directional hearing in cod (Gadus morrhua L.) J Comp Physiol 98:333-344

Schuijf A, Buwalda RJA (1980) Underwater localization - a major problem in fish acoustics. In: Popper AN, Fay RR (eds). Comparative studies of hearing in vertebrates. Springer, Berlin Heidelberg New York pp 43-77

Schuijf A, Hawkins AD (1983) Acoustic distance discrimination by the cod. Nature 302:143-144

York AG (1972) Acoustic detection and attraction of tune in New Zealand waters. Indo Pac Fish Coun Proc 115:470-480 